\title{
Apresentação atípica de degeneração marginal pelúcida: relato de caso
}

\author{
Atypical presentation of pellucid marginal degeneration:case report
}

\author{
Frederico Xavier dos Santos ${ }^{1}$ \\ Sheila Mocelin ${ }^{2}$ \\ Renato Machado ${ }^{3}$ \\ Fernando Henrique Xavier dos Santos ${ }^{4}$ \\ Luciene Barbosa de Sousa ${ }^{5}$
}

\section{RESUMO}

Os autores relatam o caso de paciente jovem do sexo masculino que desenvolveu quadro de degeneração marginal pelúcida bilateral atípica, com afinamento corneal periférico de $360^{\circ}$, abordando ainda os aspectos dos mapas topográficos e os resultados obtidos com a paquimetria ultrasônica, além do tratamento adotado.

Descritores: Córnea/ultra-sonografia; Córnea/patologia; Diagnóstico diferencial; Acuidade visual; Topografia da córnea; Técnicas de diagnóstico oftalmológico; Relatos de casos [tipo de publicação]

\section{INTRODUÇÃO}

Degeneração marginal pelúcia (DMP) é afinamento não-inflamatório da periferia da córnea que, com freqüência, ocorre bilateralmente com acometimento preferencial de sua região inferior ${ }^{(1)}$. O afinamento ocorre sob a forma de faixa em crescente que mede de 1-2 mm, geralmente das 4 às $8 \mathrm{~h}^{(1)}$, localizada a 1-2 mm do limbo inferior, separada do limbo córneo-escleral por área de espessura normal, sem sinais de cicatrização, vascularização ou depósitos lipídicos ${ }^{(2)}$.

O epitélio é intacto e a área acima do afinamento pode se apresentar ectasiada $^{(3)}$. De acordo com alguns autores, o mapa topográfico de pacientes com DMP clássica demonstra como característica típica o aplanamento da córnea ao longo do eixo vertical e o encurvamento da região inferior da córnea periférica ao longo da lesão, fornecendo uma imagem característica, porém não patognomônica, em "asa de borboleta"(4).

Sua patogênese ainda permanece desconhecida, podendo estar associada a uma série de fatores, incluindo herança genética ${ }^{(5)}$.

Este artigo tem por objetivo descrever um caso atípico de DMP bilateral.

Oftalmológico de Sorocaba. Sorocaba (SP).

${ }^{2}$ Médica Oftalmologista, fellow do Setor de Córnea do Hospital Oftalmológico de Sorocaba. Sorocaba (SP).

3 Médico oftalmologista, Setor de Córnea e Doenças Externas, Hospital Oftalmológico de Sorocaba. Sorocaba (SP).

${ }^{4}$ Acadêmico do sexto ano do curso de medicina da Ponti-

fícia Universidade Católica de Sorocaba. Sorocaba (SP).

${ }_{5}^{5}$ Professora Afiliada do Departamento de Oftalmologia da UNIFESP, Doutora em Oftalmologia e Coordenadora da Residência do Hospital Oftalmológico de Sorocaba. Sorocaba (SP).

Instituição de origem: Hospital Oftalmológico de Sorocaba. Rua Nabeck Shiroma, 210 - Sorocaba (SP) CEP 18031-060

Endereço para correspondência: Frederico Xavier dos Santos. Rua Bernardo Ferraz de Almeida, 187 - Apto. 1 - Sorocaba (SP) CEP 18031-000

E-mail: fredxsantos@hotmail.com

Recebido para publicação em 19.04.2005

Versão revisada recebida em 13.07.2005

Aprovação em 20.07.2005

\section{RELATO DE CASO}

ACS, sexo masculino, 26 anos de idade, natural e procedente de São Paulo - SP, procurou assistência oftalmológica queixando-se de baixa acuidade visual progressiva em ambos os olhos há 2 anos, iniciado no olho direito. Negava dor, vermelhidão, ardor, perda súbita da visão e moscas volantes. Não apresentava doenças sistêmicas e oculares prévias. Sem antecedentes de doenças oculares na família.

Ao exame oftalmológico apresentava acuidade visual de conta dedos a 2 metros em ambos os olhos com piora subjetiva no OD.

À biomicroscopia evidenciava-se olho calmo, com importante afinamento periférico bilateral de $360^{\circ}$, mais acentuado na região lateral do $\mathrm{OD}$ e região medial inferior do OE, além de moderado afinamento da região central da córnea 
com ectasia importante (Figuras 1 e 2). No OE observava-se ainda área de opacidade na região medial superior.

O epitélio apresentava-se íntegro por toda córnea, incluindo a área de afinamento, sem a presença de cicatrizes, vascularização periférica e depósitos lipídicos.

Não foi observado cone central, estrias de Vogt ou anel de Fleischer. Apresentava reflexos pupilares (direto e consensual) sem alterações, a tonometria de aplanação no OD era de $11 \mathrm{mmHg}$ e no OE de $8 \mathrm{mmHg}$. No exame de mapeamento de retina não foram observadas alterações.

Frente aos dados fornecidos pelo exame oftalmológico solicitamos paquimetria ultra-sônica para ambos os olhos que confirmou a suspeita clínica, mostrando área de afinamento corneal periférico importante e afinamento central moderado (Figura 3). O mapa topográfico revelou astigmatismo irregular e assimétrico importante em ambos os olhos formando imagem em "asa de borboleta", melhor observado no OD. Apresentava ceratometria no OD de 58,91 X 47,13 a $100^{\circ}$ e no OE de 57,87 $\mathrm{X} 46,53$ a $70^{\circ}$ (Figura 4 ).

Após correlação do exame físico com os resultados dos exames complementares ficou firmado o diagnóstico de DMP atípico com afinamento periférico de $360^{\circ}$.

Como tratamento inicial foi proposto teste de lentes de contato, na tentativa de melhora da acuidade visual, entretanto, devido a importante alteração da curvatura corneal, não houve estabilização das lentes testadas. Devido ao insucesso da adaptação das lentes, foi proposto tratamento cirúrgico com a realização de transplante lamelar anterior, superficial, manual e anelar, com o intuito de proporcionar a regularização da superfície corneal para posterior adaptação de lentes de contato.
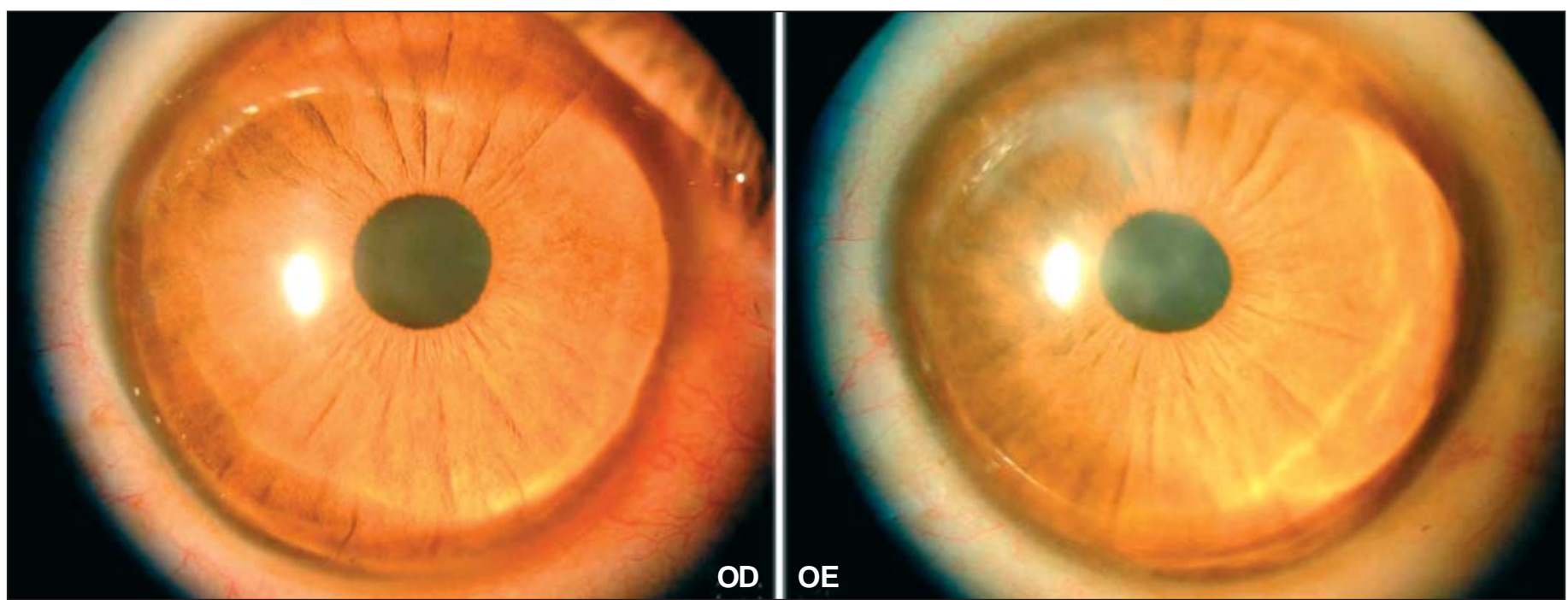

Figura 1 - Exame biomicroscópico, evidenciando afinamento corneano periférico importante, com opacidade corneana superior em OE
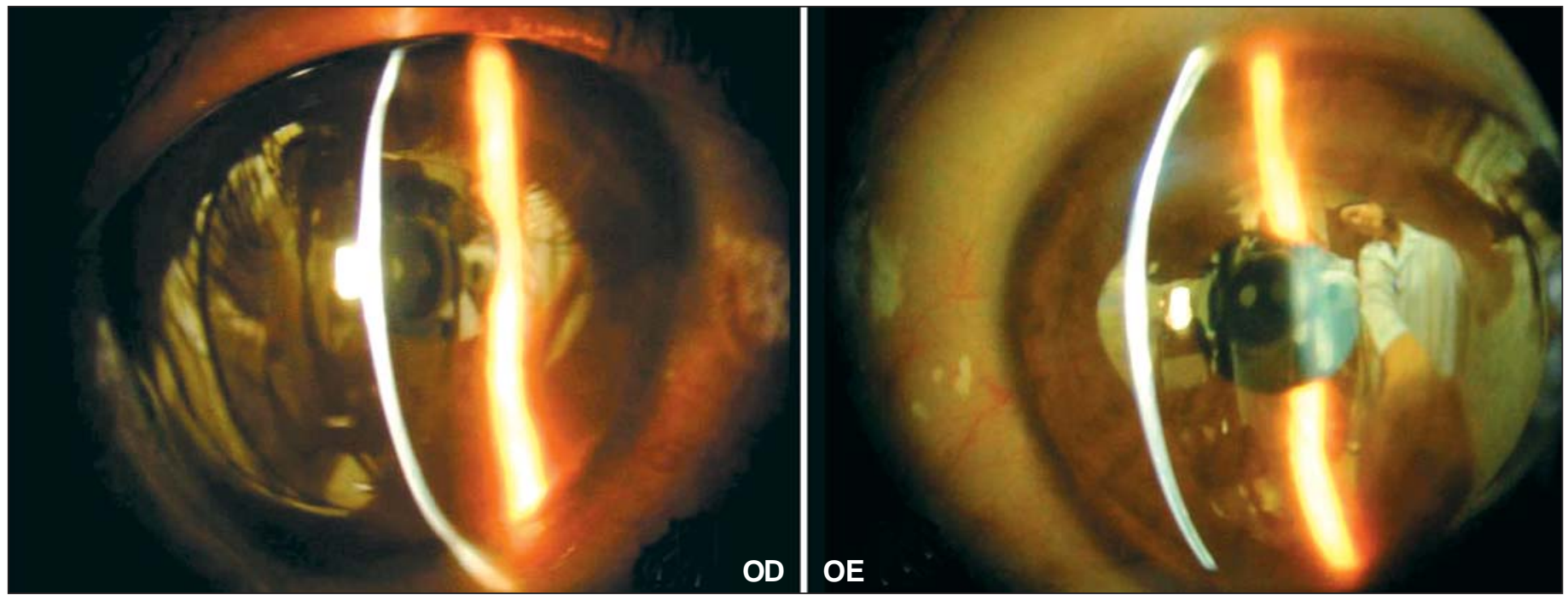

Figura 2 - Corte óptico mostrando afinamento corneano periférico 
Paciente encontra-se no segundo mês de período pósoperatório de transplante lamelar anelar do OD, com acuidade visual sem correção de 0,1 (medido em tabela de Snellen a $6 \mathrm{~m}$ ) e com ceratometria de 49,34 X 47,62 a $74^{\circ}$, evidenciando o importante aplanamento da curvatura corneana.

\section{DISCUSSÃO}

A degeneração marginal pelúcia é uma condição rara que acomete preferencialmente paciente do sexo masculino, com idade entre $20 \mathrm{e} 40$ anos $^{(3)}$. Usualmente é bilateral, porém existem alguns relatos de casos com envolvimento unilateral ${ }^{(6)}$.

Apresenta-se como importante diagnóstico diferencial nos quadros de ceratocone, ceratoglobo, degeneração marginal de Terrien, úlcera de Mooren, rosácea ocular e outras causas de afinamentos periféricos como as causadas por doenças sistêmicas auto-imunes ${ }^{(6)}$.

Apesar da forma clássica da DMP (afinamento corneal inferior) ser a mais comum, existem relatos que mostram esta doença de aspectos diferentes, resultantes do acometimento de outras regiões da periferia corneal, que por vezes faz sua evolução clínica se confundir com alguns de seus diagnósticos diferenciais.

A literatura nos mostra relatos de casos de DMP com envolvimento da periferia superior da córnea, sendo que a região inferior estava preservada ${ }^{(1,7-9)}$. Este envolvimento pode ocorrer em qualquer quadrante da córnea. Um estudo reportou as características topográficas de olhos com DMP

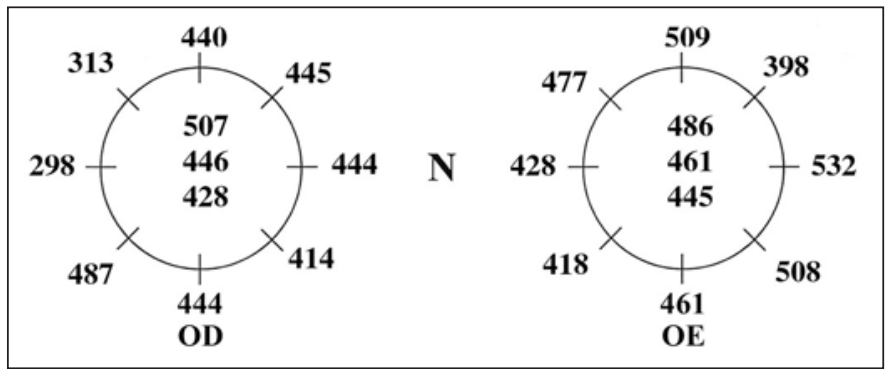

Figura 3 - Paquimetria ultra-sônica mostrando afinamento corneano difuso, principalmente em região periférica

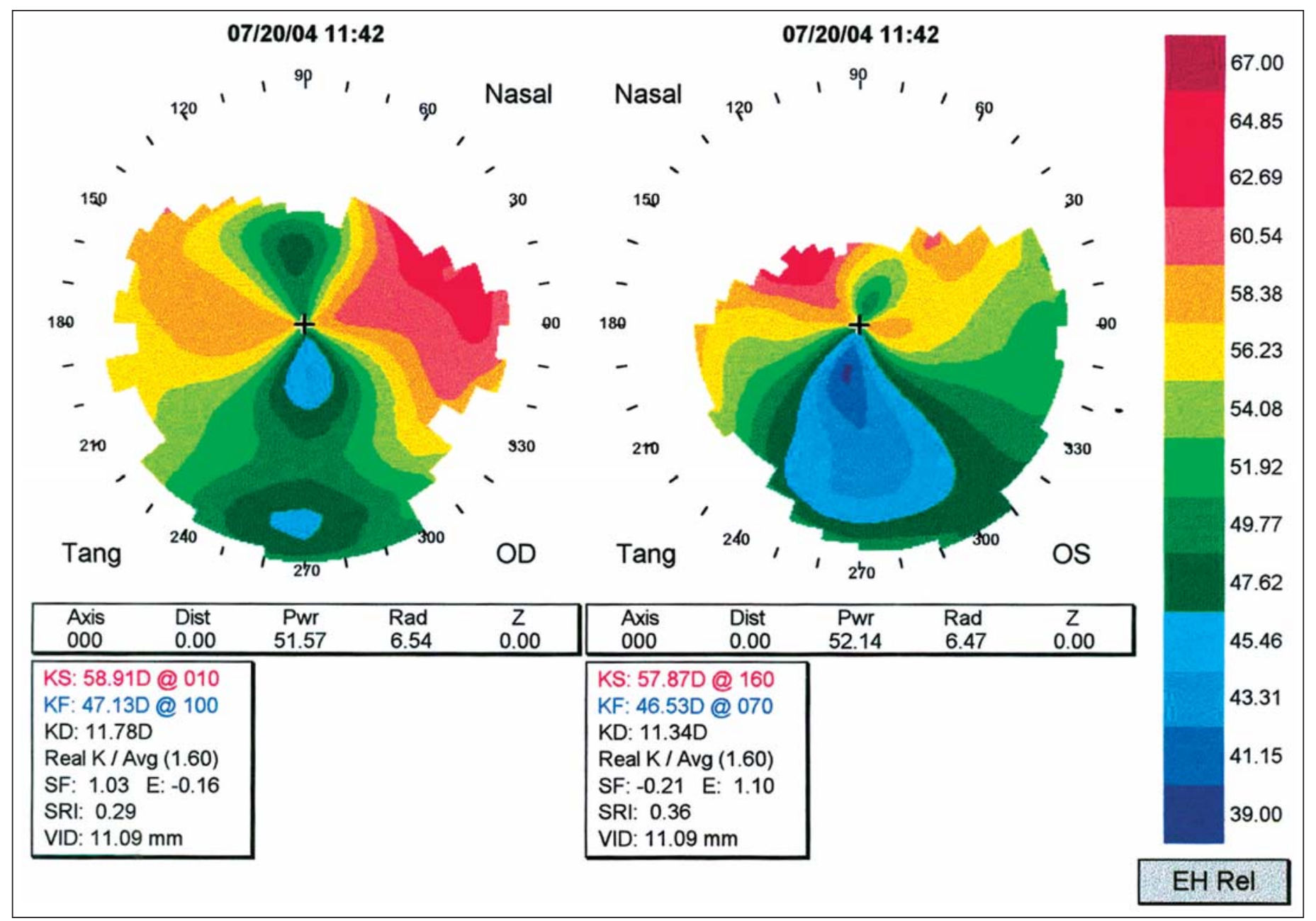

Figura 4 - Exame topográfico evidenciando astigmatismo irregular em ambos os olhos 
em locais atípicos, incluindo um caso de DMP com acometimento medial e três casos com afinamento superior. Cerca de $15 \%$ dos olhos apresentavam apenas DMP com comprometimento da periferia superior ${ }^{(8)}$.

Como a etiopatogenia da DMP ainda permanece obscura, alguns estudos sugerem que esta condição mantenha íntima relação com a hereditariedade ${ }^{(5)}$. Relatos de membros de uma família com diferentes ectasias corneais além de relatos de pacientes com características de ceratocone e DMP em um mesmo olho sugerem que as ectasias corneanas possam representar aspectos clínicos distintos de um mesmo problema ${ }^{(10)}$.

Outro trabalho descreve as alterações topográficas em cinco membros assintomáticos de uma família de um paciente com DMP clássica, sendo observado que um membro apresentava padrão inicial de DMP clássica, outro apresentava mapa topográfico com encurvamento corneal central em ambos os olhos sugestivo de ectasia corneal, um membro com padrão de ceratocone em fase inicial, um membro com padrão "bow-tie" em ambos os olhos e por fim um membro com encurvamento anormal envolvendo toda a córnea ${ }^{(11)}$. Esses achados reforçam a idéia de que as ectasias corneais possam apresentar fatores etiológicos comuns, com o envolvimento da herança genética com diferentes padrões de penetrância genética.

O relato do caso de um paciente com características clínicas e topográficas de DMP clássica em um dos olhos e no outro quadro de ceratoglobo com importante encurvamento da região periférica inferior da córnea avaliado por exame topográfico, sugere que devido à alteração topográfica, a DMP poderia preceder o desenvolvimento do ceratoglobo, enfatizando que ambas doenças pertencem ao mesmo espectro de afinamentos corneais não-inflamatórios ${ }^{(12)}$.

Concluindo, a DMP é uma doença que pode se apresentar de várias formas com o acometimento de todos os quadrantes da córnea e que por vezes sua evolução pode se confundir com outras doenças não-inflamatórias que se manifestam por afinamento corneal, como ceratocone e ceratoglobo. Este trabalho teve o objetivo de relatar mais uma forma atípica de desenvolvimento de DMP com afinamento da periferia corneal em $360^{\circ}$, contribuindo assim para aumentar as formas atípicas de DMP e enfatizar a importância de se incluir a DMP como diagnóstico diferencial para os afinamentos corneais periféricos de uma maneira geral e não apenas naqueles com comprometimento da região inferior da córnea.

\section{AGRADECIMENTO}

Ao Dr. Paulo Elias Correa Dantas por ter realizado a revisão final deste artigo, contribuindo de maneira ímpar para a melhora deste.

\section{ABSTRACT}

The authors report an unusual clinical presentation of pellucid marginal degeneration with $360^{\circ}$ peripheral corneal thinning diagnosed in a younger male patient. We discuss the findings of topographic maps, ultrasound pachymetry and the proposed treatment.

Keywords: Cornea/ultrasonography; Cornea/pathology; Diagnosis, differential; Visual acuity; Corneal topography; Diagnostic techniques, ophthalmological; Case reports [publication type]

\section{REFERÊNCIAS}

1. Bower KS, Dhaliwal DK, Barnhorst DA Jr., Warnicke J. Pellucid marginal degeneration with superior corneal thinning. Cornea. 1997;16(4):483-5.

2. Taglia DP, Sugar J. Superior pellucid marginal degeneration with hydrops. Arch Ophthalmol. 1997;115(2):274-5.

3. Sridhar MS, Mahesh S, Bansal AK. Pellucid marginal corneal degeneration. Ophthalmology. 2004;111(6):1102-7.

4. Maguire RJ, Klyce SD, McDonald MB, Kaufman HE. Corneal topography of pellucid marginal degeneration. Ophthalmology. 1987;94(5):519-24.

5. Krachmer JH, Feder RS, Belin MW. Keratoconus and related non inflammatory corneal thinning disorders. Surv Ophthalmol. 1984;28(4):293-322.

6. Biswas S, Brahma A, Tromans C, Ridgway A. Management of pellucid marginal corneal degeneration. Eye. 2000;14(Pt 4):629-34.

7. Orlin SE, Sulewski ME. Spontaneous corneal perfuration in pellucid marginal degeneration. CLAO J. 1998;24(3):186-7.

8. Rao SK, Fogla R, Padmanabhan P, Sitalakshmi G. Corneal topography in atipical pellucid marginal degeneration. Cornea. 1999;18(3):265-72.

9. Cameron JA, Mahmood MA. Superior corneal thinning with pellucid marginal corneal degeneration. Am J Ophthalmol. 1990;109(4):486-7.

10. Kayazawa F, Nishimura K, Kodama Y, Tsuji T, Itoi M. Keratoconus with pellucid marginal corneal degeneration. Arch Ophthalmol. 1984;102(6):895-6.

11. Santo MR, Bechara SJ, Kara-José N. Corneal topography in asymptomatic family members of a patient with pellucid marginal degeneration. Am J Ophthalmol. 1999;127(2):205-7.

12. Karabatsas $\mathrm{CH}$, Cook DS. Topographic analysis in pellucid marginal corneal degeneration and keratoglobus. Eye. 1996;10(Pt 4):451-5. 